\title{
Misdiagnosis of Rectus Abdominis Abscess Owing to Delayed Contrast- Enhanced Computed Tomography
}

\author{
Yuichiro Nagase $^{1}$, Yukinori Harada ${ }^{1,2}$ \\ ${ }^{1}$ Department of Internal Medicine, Nagano Chuo Hospital, Nagano, Nagano, Japan \\ ${ }^{2}$ Department of Diagnostic and Generalist Medicine, Dokkyo Medical University Hospital, Mibu, Tochigi, Japan
}

Received: 30/11/2021

Accepted: $10 / 12 / 2021$

Published: $30 / 12 / 2021$

How to cite this article: Nagase Y, Harada Y. Misdiagnosis of rectus abdominis abscess owing to delayed contrast-enhaced computed tomography. EJCRIM 2021;8: doi:10.12890/2021_003097.

Conflicts of Interests: The authors declare there are no competing interests.

This article is licensed under a Commons Attribution Non-Commercial 4.0 License

\section{ABSTRACT}

A 77-year-old man, who was on anticoagulation, presented with a painful lump on the right abdominal wall. Laboratory tests showed slight anaemia and elevated inflammatory markers. Abdominal plain computed tomography (CT) revealed a mass in the right rectus abdominis muscle. He was admitted with a diagnosis of primary rectus abdominis haematoma. However, on the next day, the diagnosis was corrected to primary rectus abdominis abscess, following contrast-enhanced CT of the abdomen. This case illustrates the importance of considering primary rectus abdominis abscess in patients with suspected primary rectus abdominis haematoma, and contrast should be used when performing CT.

\section{LEARNING POINTS}

- Primary rectus abdominis abscess should be considered in patients with suspected primary rectus abdominis haematoma.

- To differentiate rectus abdominis abscess from rectus abdominis haematoma, contrast should be used when computed tomography is performed.

\section{KEYWORDS}

Contrast-enhanced computed tomography, primary rectus abdominis abscess, primary rectus abdominis haematoma

\section{INTRODUCTION}

Primary rectus abdominis abscess is a relatively rare condition that requires antimicrobials and drainage for treatment. However, this condition can easily be misdiagnosed as the more common primary rectus abdominis haematoma, because the two conditions have several identical contributing factors, symptoms and signs ${ }^{[1,2]}$. We report a case of primary rectus abdominis abscess which was initially misdiagnosed as primary rectus abdominis haematoma.

\section{CASE DESCRIPTION}

A 77-year-old man presented to our hospital with a painful lump on the right abdominal wall. The pain developed 10 days before the visit, and the lump appeared 6 days later after he first felt pain. The patient denied exercise or trauma, but recalled that the pain gradually developed after straining in the toilet. He was taking warfarin and clopidogrel for coronary artery disease after coronary artery bypass grafting and aortic valve replacement. He also had hypertension and diabetes mellitus. 
On examination, he was afebrile. A warm, tender mass was observed in the right lower abdomen. Laboratory tests showed a white blood cell

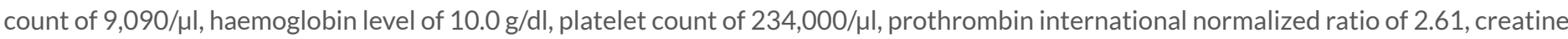
phosphokinase of $64 \mathrm{U} / \mathrm{l}$, creatinine of $0.96 \mathrm{mg} / \mathrm{dl}$, and a C-reactive protein level of $9.24 \mathrm{mg} / \mathrm{dl}$. Abdominal plain computed tomography (CT) revealed a $40 \mathrm{~mm}$ mass in the right rectus abdominis muscle (Fig. 1a). He was admitted with a diagnosis of primary rectus abdominis haematoma.

Ultrasonography was performed to evaluate the internal properties of the lump, which revealed an irregular hypoechoic mass in the rectus abdominis muscle. Considering this finding and the elevated C-reactive protein level on day two, contrast-enhanced CT of the abdomen was conducted to re-evaluate the mass. CT showed a hypointense mass with a delayed enhanced rim and fat stranding around the mass in the right rectus abdominis muscle (Fig. 1b). Streptococcus constellatus, Citrobacter freundii, Haemophilus aphrophilus and Proteus vulgaris were identified from pus drainage cultures. Additional investigations showed no evidence of infective endocarditis or other abscesses. The patient was finally diagnosed with primary rectus abdominis abscess, and treated with intravenous meropenem for 14 days, followed by oral levofloxacin for 14 days. The abscess disappeared after treatment.

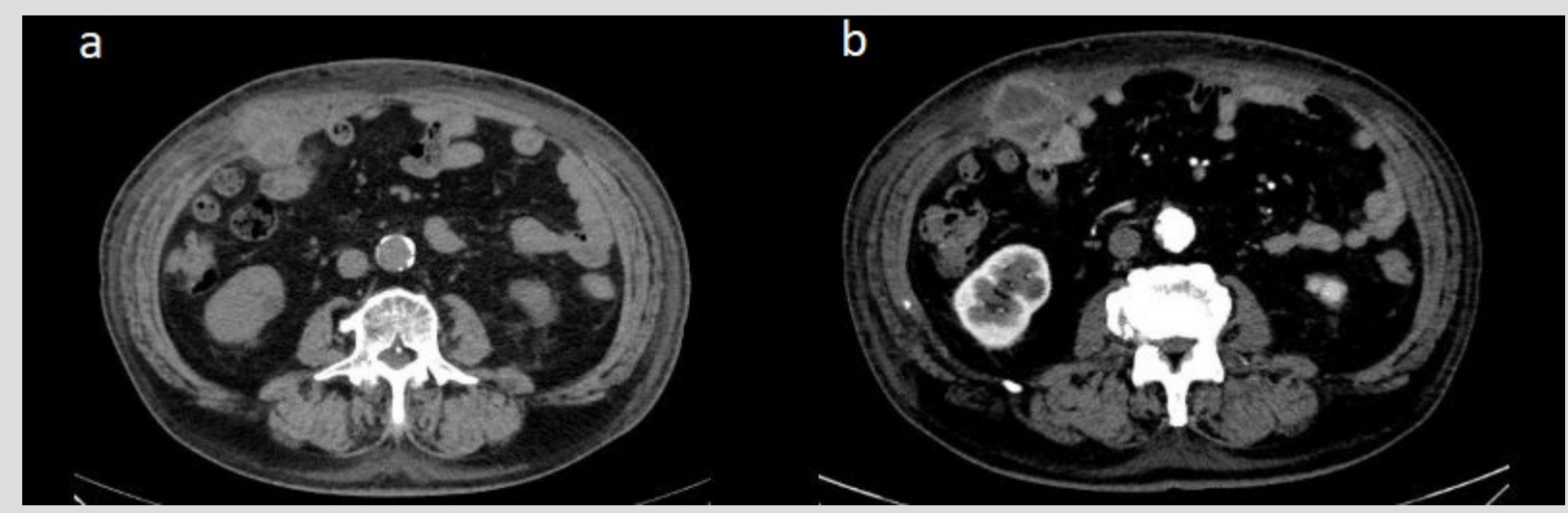

Figure 1. (a) Non-contrast $\mathrm{CT}$ of the abdomen performed on day of admission showed a $40 \mathrm{~mm}$ mass lesion in the right rectus abdominis muscle. (b) Abdominal contrast-enhanced CT on day two revealed an abscess

\section{DISCUSSION}

This case illustrates the importance of considering primary rectus abdominis abscess in patients with suspected primary rectus abdominis haematoma. Our patient exhibited risk factors for primary rectus abdominis haematoma, including old age, hypertension, and taking of anticoagulation and antiplatelet drugs. However, some observations were inconsistent, including the type of onset (subacute rather than sudden or acute) and elevation of C-reactive protein level. As reported previously, primary rectus abdominis abscess should be considered in male patients with diabetes and elevated C-reactive protein levels.

In patients suspected of primary rectus abdominis abscess, abdominal ultrasonography ${ }^{[3]}$ and contrast-enhanced CT are suitable modalities for further evaluation. However, since the quality and interpretation of ultrasonography are operator-dependent, contrast-enhanced CT is recommended. Characteristic CT findings of primary rectus abdominis abscess include diffuse oedema with cellulitis, fluid accumulation and gas-fluid levels ${ }^{[4]}$. As observed in our case, contrast should be used when performing CT of abdominal wall mass lesions so as to discriminate primary rectus abdominis abscess from primary rectus abdominis haematoma.

In conclusion, clinicians should consider primary rectus abdominis abscess in patients suspected of primary rectus abdominis haematoma, especially in males with diabetes and elevated C-reactive protein levels. Contrast should be used when performing CT to differentiate primary rectus abdominis abscess from primary rectus abdominis haematoma accurately.

\section{REFERENCES}

Smithson A, Ruiz J, Perello R, Valverde M, Ramos J, Garzo L. Diagnostic and management of spontaneous rectus sheath hematoma. Eur J Intern Med 2013;24(6):579-582. Shinohara S, Hyodo M, Ota G, Hayashi H, Sato H, Tsukahara M, et al. A primary abscess in the rectus abdominis muscle: a case report. J Abdom Emerg Med 2019;39(5):863-866. Weiner CI, Diaconis JN. Primary abdominal wall abscess diagnosed by ultrasound. Arch Surg 1975;110(3):341-342.

Goodman P, Raval B. CT of the abdominal wall. AJR Am J Roentgenol 1990;154(6):1207-1211. 